\title{
Stroke mimics transported by emergency medical services to a comprehensive stroke center: The magnitude of the problem
}

Joel Neves Briard $\mathrm{MD}^{1}$, Rahel T. Zewude BSc ${ }^{2}$, Mahesh P. Kate $\mathrm{MD}^{2}$, Brian H. Rowe MD $\mathrm{MSc}^{3}$, Brian Buck MD MSc${ }^{2}$, Ken Butcher MD PhD ${ }^{2}$, Laura C. Gioia MD MSc${ }^{1}$

This article has been published in a revised form in the Journal of Stroke and Cerebrovascular Diseases: https://doi.org/10.1016/j.jstrokecerebrovasdis.2018.05.046

(C) 2018. This manuscript version is made available under the CC-BY-NC-ND 4.0

license http://creativecommons.org/licenses/by-nc-nd/4.0/

Submitted: 7 April 2018. Accepted: 28 May 2018.

\section{Affiliations :}

1. Department of Neurosciences, Université de Montréal, Quebec, Canada \& Centre de recherche du Centre hospitalier de l'Université de Montréal, Quebec, Canada

2. Division of Neurology, University of Alberta, Alberta, Canada

3. Department of Emergency Medicine and School of Public Health, University of Alberta, Alberta, Canada

Citation: Neves Briard J, Zewude RT, Kate MP, Rowe BH, Buck B, Butcher K, Gioia LC. Stroke mimics transported by emergency medical services to a comprehensive stroke center: The magnitude of the problem. J Stroke Cerebrovasc Dis. 2018;27(10):2738-45

Short Title: Stroke Mimics Transported by EMS

Abstract (words): $249 \quad$ Text (words): 3029

Figures: 1

Tables: 2

Corresponding author information:

Laura C. Gioia MD MSc

Centre Hospitalier de l'Université de Montréal (CHUM)

Pavillon C, 13e Nord

1051 rue Sanguinet, porte C.13.6121

Montreal, Quebec, Canada

$\mathrm{H} 2 \mathrm{X} 0 \mathrm{C} 1$

Email: laura.gioia@umontreal.ca 


\section{Abstract}

Background: Despite the use of validated prehospital stroke screens, stroke mimics are frequent among patients transported by Emergency Medical Services to the Emergency Department. We aimed to describe the frequency and characteristics of neurological and non-neurological mimics transported to a comprehensive stroke center for acute stroke evaluation.

Methods: This was a retrospective analysis of a database consisting of all consecutive patients with suspected stroke transported to the Emergency Department of a comprehensive stroke center during an 18-month period. Hospital charts and neuroimaging were utilized to adjudicate the final diagnosis (acute stroke, stroke mimic, and specific underlying diagnoses).

Results: 950 patients were transported with suspected stroke, among whom 405 (42.6\%) were stroke mimics (age 66.9 \pm 17.1 years; 54\% male). Neurological mimics were diagnosed in 223 (55.1\%) patients and mimics were non-neurological in 182 . The most common neurological diagnoses were seizures $(19.7 \%)$, migraines $(18.8 \%)$ and peripheral neuropathies $(11.2 \%)$. Cardiovascular (14.6\%) and psychiatric (11.9\%) diagnoses were common non-neurological mimics. Patients with neurological mimic were younger $(64.1 \pm 17.3$ years vs. $70.5 \pm 16.1$ years, $\mathrm{p}<0.001)$ and had less vascular risk factors than non-neurological mimics. The proportion of nonneurological mimics remained high (38\%) despite the use of a prehospital identification scale.

Conclusion: Stroke mimics are common among patients transported by Emergency Medical Services to a comprehensive stroke center for suspected stroke, with a considerable proportion being non-neurological in origin. Studies refining triage and transport of suspected acute stroke may be warranted to minimize the number of mimics transported by to a comprehensive stroke center for acute stroke evaluation. 


\section{Introduction}

Rapid triage of acute stroke patients by Emergency Medical Services (EMS) is associated with reduced transport delays to the Emergency Department (ED) for acute stroke evaluation (1), higher use of thrombolytic therapy (2), and improved outcomes (3). EMS protocols diverting acute stroke patients from primary care hospitals to comprehensive stroke centers (CSC) with endovascular thrombectomy capabilities are increasingly popular in patients with suspected severe stroke, although robust evidence supporting this practice is currently lacking (4). Mass bypass of primary care sites to CSC is likely associated with several patients ineligible to endovascular treatment being subjected to delays in hospital care, including intravenous tissue plasminogen activator (tPA) administration.

Validated prehospital stroke identification scales are widely used by EMS professionals to detect acute stroke in the field (5-10). Prehospital identification of acute stroke remains challenging nonetheless as there are several non-stroke conditions ("stroke mimics") that share similar clinical features. As a result, prehospital stroke scales all tend to suffer from a relatively poor specificity $(24-99 \%)$ (11). Previous studies have reported that 10-44\% of EMS-transported suspected stroke patients are ultimately diagnosed with a stroke mimic in the ED $(9,12-15)$, yet little information exists regarding prehospital stroke mimics, particularly those transported to CSC. Prehospital triage of stroke mimics (including EMS diversion of primary hospitals to CSC) may be associated with considerable increases in patient volume, risks, and resource utilization in CSC (12). Despite this, little information exists regarding mimics in the prehospital setting. The aim of this study was to describe the prevalence and clinical characteristics of stroke mimics transported by EMS for acute stroke evaluation to a Canadian regional CSC.

\section{Methods}




\section{Study Design}

The methods of this study have been described previously (16). Briefly, this was a secondary, cross-sectional, observational study of a prospectively-maintained centralized EMS database of consecutive patients with suspected stroke transported by EMS to the ED of the University of Alberta Hospital (UAH), in Edmonton, Canada during an 18-month period (January $1,2012$, to July 31,2013$)$. The UAH ED manages approximately 77,000 patients per year, is staffed by full time certified emergency physicians, and functions as an academic teaching hospital for the University of Alberta. The UAH is staffed 24-hours per day by a stroke service with a dedicated on-call stroke fellow and neurologist.

The unified EMS service for the city of Edmonton, overseen by Alberta Health Services, attends to more than 157,000 events annually and transports patients to one of 13 provincially operated Edmonton Zone EDs. The UAH is a tertiary health care center and one of two CSC within the Edmonton Zone, receiving the majority of patients transported by EMS for suspected stroke and admitting 1,000 to 1,200 stroke patients annually. At the time of this study, EMS did not bypass primary hospitals for the UAH in cases of suspected stroke.

During a 9-1-1 call for suspected stroke, the emergency dispatcher is trained to employ a stroke-specific interview algorithm to assign a Medical Priority Dispatch System event code for suspected stroke. This event code is subsequently communicated to EMS providers responding to the call. The Cincinnati Prehospital Stroke Screen (CPSS) (7) was used on the field by EMS providers to determine a primary paramedic impression of acute stroke. Dispatch and individual electronic patient health care reports data were then prospectively uploaded to a centralized provincial EMS database housed securely within Alberta Health Services. The research protocol 
of this study was approved for waiver of consent by the local institutional Human Research Ethics Review Board (University of Alberta).

\section{Study Population and Data Collection}

Eligible study participants were identified within the centralized Alberta Health Services database by "suspected stroke" dispatch event and/or paramedic primary impression of acute stroke. Once identified, data from individual electronic patient health care reports data were extracted and linked to ED records through the Emergency Department Information Systems. Electronic patient health care report data were also linked to in-hospital patient charts and neuroimaging to determine the final diagnosis of ischemic stroke, transient ischemic attack (TIA), intracerebral hemorrage $(\mathrm{ICH})$ or stroke mimic, as per the treating physician's discharge diagnosis.

\section{Measures}

Stroke mimics were defined after chart and neuroimaging review as any discharge diagnosis - other than acute stroke - responsible for the initial symptoms that prompted EMS transport for suspected stroke. Mimics were classified by the first author as being neurological or nonneurological in origin, and then further divided into subcategories (neurological: seizure, delirium, dementia, neoplasm, vertigo, peripheral neuropathy, migraine, weakness of undetermined origin, and movement disorder; non-neurological: cardiovascular, metabolic, gastroenterological, psychiatric and infectious).

Participant baseline characteristics - including age, sex, smoking, alcohol use, history of hypertension, type 2 diabetes, dyslipidemia, coronary artery disease, atrial fibrillation or flutter, congestive heart failure, cognitive disorder, prior ischemic stroke, ICH or TIA - as well as 
paramedic primary impression, prehospital systolic blood pressure, prehospital Glasgow Coma Scale score, reperfusion with tPA or endovascular treatment, and destination from the ED (discharge home vs hospital admission, with admission length) were extracted for all eligible patients.

\section{Statistical Analyses}

Continuous variables are reported as means and standard deviations (SD) or medians and interquartile ranges (IQR), as appropriate. Dichotomous variables are reported as proportions. Differences in baseline characteristics between neurological and non-neurological mimics were assessed using independent t-tests (parametric data), Mann-Whitney tests (nonparametric) or chisquare test, as appropriate. No specific sample size was determined for this study; however, the sample provides $95 \%$ confidence intervals $(\mathrm{CI})$ with a precision of $+/-3 \%$ for mid-point estimates.

\section{Results}

A total of 950 eligible patients meeting study inclusion criteria were identified. Among these, 545 (57.3\%; CI: 54.0-60.4) were diagnosed with acute stroke (367 (38.6\%; CI: 35.5-41.8) ischemic stroke, 117 (12.3\%; CI: 10.3-15.3) transient ischemic attack, and 61 (6.3\%; CI: 4.1-8.1) acute intracerebral hemorrage) and 405 (42.6\%; CI: 39.5-45.8) patients were diagnosed with a stroke mimic. The mean age of all stroke mimics was $66.9 \pm 17.1$ years, and $54 \%$ of patients were male. Compared to acute stroke patients, patients with stroke mimics were younger, and had lower rates of vascular risk factors (such as hypertension, atrial fibrillarion, dyslipidemia and coronary artery disease) than patients with acute stroke (Table 1). Nonetheless, baseline vascular risk factors were frequently found in patients with stroke mimics (hypertension 53\%, type 2 diabetes 28\%, dyslipidemia 33\%, Table 1). 
Neurological and Non-Neurological Stroke Mimics

Mimics considered neurological in origin occurred in 223 (55.1\%; CI: 50.1-60.0) patients and non-neurological mimics were diagnosed in 182 (44.9\%; CI: 40.0-49.9). Patients with neurological mimics were younger $(64.1 \pm 17.3$ years vs. $70.5 \pm 16.1$ years, $p<0.001)$ and had slightly higher prehospital systolic blood pressures $(147.8 \pm 24.2 \mathrm{mmHg}$ vs. $141.2 \pm 26.2 \mathrm{mmHg}$, $\mathrm{p}=0.01$ ) than non-neurological mimics, respectively (Table 1). Median (IQR) prehospital Glasgow Coma Scale scores were similar between groups (15 (1) vs. 15 (1), $\mathrm{p}=0.26$ ). Patients with nonneurological mimics more commonly exhibited previous diagnoses of hypertension (64\% vs 44\%), type 2 diabetes (34\% vs 24\%), alcohol use (20\% vs 9\%) and congestive heart failure (10\% vs 4\%) and than neurological mimics $(\mathrm{p} \leq 0.01)$. There were no others significant differences between the two groups regarding other vascular risk factors. The only two mimic patients to have received tPA were suffering from neurological stroke mimics (specifically, one seizure and one weakness of undetermined origin). No stroke mimic patient was sent to the endovascular lab. Patient destination from the ED was similar between neurological and non-neurological mimics, with approximately $40 \%$ of patients in both groups being admitted to the hospital $(\mathrm{p}=0.81)$. Admitted non-neurological mimic patients required longer courses of hospitalisation than admitted neurological mimic patients (5 days (IQR 11) vs 2 days (IQR 8), $\mathrm{p}=0.04)$.

\section{Stroke Mimics by Specific Diagnosis}

Table 2 describes the prevalence of neurological and non-neurological mimic final diagnoses. Cardiovascular $(n=59,15.9 \%)$, psychiatric $(n=48,11.9 \%)$, and infectious $(n=36,8.9 \%)$ diagnoses were the most common causes of non-neurological stroke mimics (Figure 1A). Nearly 
half of cardiovascular mimic cases were syncope/presyncope $(n=29,7 \%)$. Among neurological mimics, the most common final diagnoses were seizures $(n=44,19.7 \%)$, migraines $(n=42,18.8 \%)$, and peripheral neuropathies $(\mathrm{n}=25,11.2 \%)$ (Figure 1B).

\section{Primary EMS Impression of Stroke}

A primary EMS impression of acute stroke was specified in 210 out of the 405 mimic patients (51.8\%). The remaining 195 patients (48.1\%), although flagged as a suspected acute stroke by emergency medical dispatch, had an alternate primary EMS impression. Non-neurological stroke mimics were less common but remained frequent $(38.1 \%)$ in patients with an EMS impression of stroke, compared with patients with an alternate impression (52.3\%, Table 2). Specifically, patients with an initial EMS impression of stroke had lower rates of cardiovascular (10\% vs $20 \%)$ and infectious (5\% vs $13 \%)$ pathologies, but there was a slightly higher prevalence of metabolic mimics (7\% vs 3\%).

\section{Discussion}

In an analysis of a centralized EMS database comprised of all consecutive patients transported for suspected stroke to a regional CSC, we found that stroke mimics represent a substantial number $(>40 \%)$ of patients evaluated and transported by EMS. Although the majority of stroke mimics were neurological in origin, a considerable and surprising number of stroke mimics were ultimately diagnosed with a non-neurological condition. Non-neurological mimic patients were older and more likely to have multiple vascular risk factors than neurological mimics. Furthermore, the proportion of non-neurological stroke mimics remained high despite screening with CPSS, a validated and widely implemented prehospital stroke identification scale. These 
results highlight the important challenge faced by EMS providers when identifying acute stroke in the prehospital field.

Our findings are consistent with previous studies showing that stroke mimics are common among patients evaluated in the ED for suspected stroke $(9,12-15)$. In previous studies, stroke mimicking pathologies were more prevalent in suspected stroke patients with the following characteristics: young age ( $<50$ years), female sex, absence of vascular risk factors, particularly atrial fibrillation, and a history of epilepsy or psychiatric illness $(15,17,18)$. In our cohort, many of these mimic characteristics - younger age and absence of alcohol use, hypertension, type 2 diabetes and congestive heart failure - were common in stroke mimics, particularly neurological mimics. To our knowledge, our study is the first to stratify stroke mimic pathologies in this detail and identify differences in neurological and non-neurological stroke mimic patient characteristics.

The overtriage of stroke mimics to CSC may have negative impacts on both patients and the health care system. Mass bypass of primary care sites to CSC is likely associated with several patients ineligible to endovascular treatment being subjected to delays in hospital care, particularly intravenous tPA administration. Moreover, overtriage of stroke mimics to CSC results in overactivation of stroke protocols, requiring mobilization of a number of healthcare professionals involved in acute stroke care (emergency physicians, triage and emergency nursing, stroke fellows and neurologists, radiology technicians and neurointerventionalists). Finally, admitted mimic patients are often subjected to extensive work-ups (19), which may expose them to unnecessary radiation or interventions, and represent significant costs to the system.

False diagnosis in the prehospital setting is not unique to acute stroke management. Studies have shown that $8-25 \%$ of cardiac catherterization lab activations following prehospital notification for acute ST-elevation myocardial infarction (STEMI) result from false-positive EMS screening 
(20-22). These false-positive cases for STEMI are regarded as acceptable, particularly since EMS activation expedites care and translates into shorter door-to-balloon delays than ED activation (23, 24). Considering the significant burden of ischemic stroke on the individual and society in association with the proven benefit of early stroke detection and overwhelming efficacy of acute reperfusion therapies, a higher false positive rate may be acceptable at a societal level. Conversely, overactivation of stroke teams and management of a high proportion of stroke mimics may put considerable strain on the health system from a resource utilization perspective. Overactivation of acute stroke may also be associated with negative outcomes for other ED patients for whom care is delayed. An increase in the length of stay in ED patients overall has been described in designated trauma centers with trauma activations (25-27). As the volume of patients eligible for endovascular intervention rapidly evolves $(28,29)$, and as stroke mimic incidence is projected to increase and possibly surpass acute stroke incidence in the future (19), it will be increasingly important to address the problem of overtriage of stroke mimics to CSC.

Several prehospital stroke identification scales have been developed to improve acute stroke detection in the out-of-hospital setting. Overall, these scales are associated with reasonably high sensitivity and low specificity for acute stroke diagnosis (10). The Los Angeles Prehospital Stroke Screen (LAPSS), the only scale that incorporates elements suggestive of stroke mimics (such as seizure history), has been shown to be a more reliable and consistent prehospital scale for acute stroke diagnosis when compared to other scales (6).

Other in-hospital scales have been developed to improve stroke mimic detection in the ED. The Recognition of Stroke in the Emergency Room (ROSIER) scale was developed for use by emergency physicians on all suspected stroke patients prior to neuroimaging (30). The presence of focal neurological signs (e.g., face, arm or leg weakness; speech disturbance; visual field defect) 
were scored favorably for acute stroke, while the presence of seizures, confusion, or loss of consciousness were scored unfavourably (loss of points on the scale). A ROSIER score $\geq 1$ favoured the diagnosis of stroke with high diagnostic accuracy (92\%). A second scale, the FABS, was developed in order to help stratify the need for advanced neuroimaging (MRI) in suspected stroke patients with a negative head CT scan in the ED (31). In the presence of three or more of the scale elements (absence of facial droop, age $<50$ years old, absence of atrial fibrillation, $\mathrm{SBP}<150 \mathrm{mmHg}$, presence of isolated sensory deficit, or seizure history), the sensitivity (90\%) and specificity (91\%) were high for stroke mimic. Indeed, our findings are in line with the elements predictive of stroke mimics in the FABS scale such the absence of atrial fibrillation, history of seizure and lower blood pressure. Given the absence of clinical information regarding the individual items of the CPSS that triggered prehospital stroke activation in our study, we were not able to calculate FABS score in our patient population.

Our findings suggest that prehospital stroke identification scales are associated with a high rate of stroke mimics, with nearly half being non-neurological in origin. The inclusion of clinical elements suggestive of stroke mimics (e.g., syncope, seizure) may help to decrease the rate of stroke mimics transported to CSC for acute stroke evaluation, but whether these tools should be employed in the EMS setting remains unknown. Future prospective studies are warranted to assess whether the use of newly-developped prehospital scale incorporating elements predictive of stroke mimics in the field is associated with fewer stroke mimics transported to ED. An alternative approach could be a combined use of a sensitive EMS screening tool to trigger transport for acute stroke followed by a second, more specific scale in order to grade stroke severity and help minimize stroke mimics when considering EMS diversion directly to CSC, but future studies are needed. Nonetheless, both approaches would require a systems-wide analysis of all patients transported for 
suspected stroke by EMS to primary and comprehensive stroke centers in order to determine the best approach.

\section{Limitations}

There are a number of limitations to our study. First, this was a retrospective study and is subject to confounding. Patient data, however, was prospectively collected and uploaded to a centralized database which may help to limit bias. Second, inclusion of all patients with suspected stroke by dispatch code and/or EMS evaluation likely resulted in an overestimation of the proportion of stroke mimics transported to the ED. We chose to include all consecutive patients evaluated in the field for suspected stroke patients in order to better characterize stroke mimics in the field as well as determine whether EMS impression of stroke was associated with lower rates of non-neurological mimics transported to ED. Moreover, in a recent prospective field validation of the Cincinnati Stroke Triage Assessment Tool, 44\% of CPSS positive patients suffered from a stroke mimic, so our reported $43 \%$ prevalence may not be significantly overestimated (9). Since dispatcher recognition of stroke is associated with a poor positive predictive value (32), our study results may not be generalizable to regions that rely solely on paramedic identification of stroke using a prehospital stroke scale. Fourth, no adjustment was made for multiple statistical testing; however, the differences are large so the statistical results are robust. Finally, the study was limited to one Canadian academic centre, and the generalizability of these results warrants further scrutiny.

\section{Conclusion}

Stroke mimics are common and heterogenous in nature among patients transported by EMS to the $\mathrm{ED}$ of a $\mathrm{CSC}$ for acute stroke evaluation, with a substantial number being non-neurological 
in origin. Positive prehospital stroke identification by EMS may decrease the rate of nonneurological stroke mimics; however, proportions remain high. Utilization of prehospital scales incorporating elements suggestive of stroke mimics (such as syncope, seizures) may improve field detection and decrease transport of stroke mimics to $\mathrm{CSC}$, particularly in an increasingly implemented model of EMS diversion to CSC, but prospective studies are needed.

\section{Author Contributions}

JNB: designed and conceptualized the study, performed analysis and interpretation of the data and drafted the manuscript. RTZ: collected data, and revised the manuscript. MPK: designed and conceptualized the primary study and revised the manuscript. BR: designed and conceptualized the primary study and revised the manuscript. BB: performed analysis and interpretation of the data and revised the manuscript. KB: designed and conceptualized the primary study and revised the manuscript. LCG: designed and conceptualized the study, performed analysis and interpretation of the data and revised the manuscript.

\section{Sources of Funding}

KSB holds a Canada Research Chair in Cerebrovascular Disease, a Heart and Stroke Foundation of Alberta Professorship in Stroke Medicine and a New Investigator Award from Alberta Innovates Health Solutions. LCG and MK are supported by Clinical Research Fellowship bursaries from the Alberta Innovates Health Solutions. RZT received by a Summer Studentship Award from Alberta Innovates Health Solutions. BHR is supported by CIHR as a Tier I Canada Research Chair in Evidence-based Emergency Medicine through the Government of Canada (Ottawa, ON) and is the Scientific Director of the Institute of Circulatory and Respiratory Health (ICRH) at CIHR. The funding agencies take no responsibility for the conduct, results and interpretations of findings reported. 


\section{Disclosure}

The authors report no conflicts of interest. 


\section{References}

1. Caceres JA, Adil MM, Jadhav V, Chaudhry SA, Pawar S, Rodriguez GJ, et al. Diagnosis of stroke by emergency medical dispatchers and its impact on the prehospital care of patients. $J$ Stroke Cerebrovasc Dis. 2013;22(8):e610-4.

2. McKinney JS, Mylavarapu K, Lane J, Roberts V, Ohman-Strickland P, Merlin MA. Hospital prenotification of stroke patients by emergency medical services improves stroke time targets. J Stroke Cerebrovasc Dis. 2013;22(2):113-8.

3. Pérez de la Ossa N, Sanchez-Ojanguren J, Palomeras E, Millan M, Arenillas JF, Dorado $\mathrm{L}$, et al. Influence of the stroke code activation source on the outcome of acute ischemic stroke patients. Neurology. 2008;70(15):1238-43.

4. Southerland AM, Johnston KC, Molina CA, Selim MH, Kamal N, Goyal M. Suspected Large Vessel Occlusion: Should Emergency Medical Services Transport to the Nearest Primary Stroke Center or Bypass to a Comprehensive Stroke Center With Endovascular Capabilities? Stroke. 2016;47:1965-7.

5. Bray JE, Martin J, Cooper G, Barger B, Bernard S, Bladin C. Paramedic Identification of Stroke: Community Validation of the Melbourne Ambulance Stroke Screen. Cerebrovascular Diseases. 2005;20(1):28-33.

6. Kidwell CS, Starkman S, Eckstein M, Weems K, Saver JL. Identifying Stroke in the Field: Prospective Validation of the Los Angeles Prehospital Stroke Screen (LAPSS). Stroke. 2000;31:71-6.

7. Kothari RU, Pancioli A, Liu T, Brott T, Broderick JP. Cincinnati Prehospital Stroke Scale: Reproducibility and validity. Ann Emerg Med. 1999;33(4):373-8.

8. Llanes JN, Kidwell CS, Starkman S, Leary MC, Eckstein M, Saver JL. The Los Angeles Motor Scale (LAMS): A New Measure to Characterize Stroke Severity in the Field. Prehosp Emerg Care. 2009;8(1):46-50.

9. McMullan JT, Katz B, Broderick J, Schmit P, Sucharew H, Adeoye O. Prospective Prehospital Evaluation of the Cincinnati Stroke Triage Assessment Tool. Prehosp Emerg Care. 2017;21(4):481-8.

10. Pérez de la Ossa N, Carrera D, Gorchs M, Querol M, Millan M, Gomis M, et al. Design and validation of a prehospital stroke scale to predict large arterial occlusion: the rapid arterial occlusion evaluation scale. Stroke. 2014;45(1):87-91.

11. Brandler ES, Sharma MS, Sinert RH, Levine SR. Prehospital stroke scales in urban environments: a systematic review. Neurology. 2014;82:2241-9.

12. Goyal N, Male S, Al Wafai A, Bellamkonda S, Zand R. Cost burden of stroke mimics and transient ischemic attack after intravenous tissue plasminogen activator treatment. $J$ Stroke Cerebrovasc Dis. 2015;24(4):828-33.

13. Hand PJ, Kwan J, Lindley RI, Dennis MS, Wardlaw JM. Distinguishing between stroke and mimic at the bedside: the brain attack study. Stroke. 2006;37(3):769-75.

14. Sequeira D, Martin-Gill C, Kesinger MR, Thompson LR, Jovin TG, Massaro LM, et al. Characterizing Strokes and Stroke Mimics Transported by Helicopter Emergency Medical Services. Prehosp Emerg Care. 2016;20(6):723-8.

15. Vroomen PC, Buddingh MK, Luijckx GJ, De Keyser J. The incidence of stroke mimics among stroke department admissions in relation to age group. J Stroke Cerebrovasc Dis. 2008;17(6):418-22. 
16. Gioia LC, Zewude RT, Kate MP, Liss K, Rowe BH, Jeerakathil T, et al. Prehospital systolic blood pressure is higher in acute stroke compared with stroke mimics. Neurology. 2016;86(23):2146-53.

17. Khan NI, Chaku S, Goehl C, Endris L, Mueller-Luckey G, Siddiqui FM. Novel Algorithm to Help Identify Stroke Mimics. J Stroke Cerebrovasc Dis. 2018;27(3):703-8.

18. Merino JG, Luby M, Benson RT, Davis LA, Hsia AW, Latour LL, et al. Predictors of Acute Stroke Mimics in 8187 Patients Referred to a Stroke Service. J Stroke Cerebrovasc Dis. 2013;22(8):e397-e403.

19. Faiz KW, Labberton AS, Thommessen B, Ronning OM, Dahl FA, Barra M. The Burden of Stroke Mimics: Present and Future Projections. J Stroke Cerebrovasc Dis. 2017.

20. Garvey JL, Monk L, Granger CB, Studnek JR, Roettig ML, Corbett CC, et al. Rates of cardiac catheterization cancelation for ST-segment elevation myocardial infarction after activation by emergency medical services or emergency physicians: results from the North Carolina Catheterization Laboratory Activation Registry. Circulation. 2012;125(2):308-13. 21. Potter BJ, Matteau A, Mansour S, Essiambre R, Montigny M, Savoie S, et al. Performance of a new "physician-less" automated system of prehospital ST-segment elevation myocardial infarction diagnosis and catheterization laboratory activation. Am J Cardiol. 2013;112(2):156-61.

22. Squire BT, Tamayo-Sarver JH, Rashi P, Koenig W, Niemann JT. Effect of prehospital cardiac catheterization lab activation on door-to-balloon time, mortality, and false-positive activation. Prehosp Emerg Care. 2014;18(1):1-8.

23. Bradley EH, Herrin J, Wang YW, Barton BA, Webster TR, Mattera JA, et al. Strategies for Reducing the Door-to-Balloon Time in Acute Myocardial Infarction. N Engl J Med. 2006;355:2308-20.

24. Rokos IC, French WJ, Koenig WJ, Stratton SJ, Nighswonger B, Strunk B, et al. Integration of pre-hospital electrocardiograms and ST-elevation myocardial infarction receiving center (SRC) networks: impact on Door-to-Balloon times across 10 independent regions. JACC Cardiovasc Interv. 2009;2(4):339-46.

25. Arya R, Dossantos F, Ohman-Strickland P, Merlin MA. Impact of trauma activation on the ED length of stay for nontraumatic patients. Am J Emerg Med.2012;30(2):311-6.

26. Fishman PE, Shofer FS, Robey JL, Zogby KE, Reilly PM, Branas CC, et al. The impact of trauma activations on the care of emergency department patients with potential acute coronary syndromes. Ann Emerg Med. 2006;48(4):347-53.

27. Smith DC, Chapital A, Burgess Uperesa BM, Smith ER, Ho C, Ahana A. Trauma activations and their effects on non-trauma patients. J Emerg Med. 2011;41(1):90-4.

28. Albers GW, Marks MP, Kemp S, Christensen S, Tsai JP, Ortega-Gutierrez S, et al. Thrombectomy for Stroke at 6 to 16 Hours with Selection by Perfusion Imaging. $N$ Engl J Med. 2018;378(8):708-18.

29. Nogueira RG, Jadhav AP, Haussen DC, Bonafe A, Budzik RF, Bhuva P, et al. Thrombectomy 6 to 24 Hours after Stroke with a Mismatch between Deficit and Infarct. $N$ Engl J Med. 2018;378(1):11-21.

30. Nor AM, Davis J, Sen B, Shipsey D, Louw SJ, Dyker AG, et al. The Recognition of Stroke in the Emergency Room (ROSIER) scale: development and validation of a stroke recognition instrument. The Lancet Neurology. 2005;4(11):727-34.

31. Goyal M, Tsivgoulis G, Male S, Metter EJ, Iftikhar S, Kerro A, et al. FABS: An Intuitive Tool for Screening of Stroke Mimics in the Emergency Department. Stroke. 2016;47:2216-20. 
32. Oostema JA, Carle T, Talia N, Reeves M. Dispatcher Stroke Recognition Using a Stroke Screening Tool: A Systematic Review. Cerebrovasc Dis. 2016;42:370-7. 
Table 1. Baseline prehospital characteristics of neurological and non-neurological stroke mimics

\begin{tabular}{|c|c|c|c|c|c|c|}
\hline & $\begin{array}{l}\text { All strokes } \\
(\mathrm{n}=545)\end{array}$ & $\begin{array}{l}\text { All stroke } \\
\text { mimics } \\
(\mathrm{n}=405)\end{array}$ & p-value & $\begin{array}{c}\text { Neurological } \\
\text { mimics } \\
(\mathrm{n}=223)\end{array}$ & $\begin{array}{c}\text { Non-neurological } \\
\text { mimics } \\
(\mathrm{n}=182)\end{array}$ & p-value* \\
\hline \multicolumn{7}{|l|}{ Demographics } \\
\hline Age, years (mean \pm SD) & $73.0 \pm 14$ & $66.9 \pm 17.3$ & $<0.001^{\text {ब }}$ & $64.1 \pm 17.3$ & $70.5 \pm 16.1$ & $<0.001 *$ \\
\hline Sex $(\mathrm{n}, \%$ male $)$ & $268(49)$ & $217(54)$ & & $125(56)$ & $92(51)$ & 0.34 \\
\hline \multicolumn{7}{|l|}{ Baseline characteristics } \\
\hline Smoking $(\mathrm{n}, \%)$ & $134(25)$ & $76(19)$ & 0.02 ฯ & $35(16)$ & $41(23)$ & 0.05 \\
\hline Alcohol use & $55(11)$ & $58(14)$ & 0.07 & $21(9)$ & $37(20)$ & $0.001 *$ \\
\hline Hypertension & $397(73)$ & $215(53)$ & $<0.001^{\text {ף }}$ & $99(44)$ & $116(64)$ & $<0.001 *$ \\
\hline Type 2 diabetes & $129(24)$ & $115(28)$ & 0.30 & $53(24)$ & $62(34)$ & $0.01 *$ \\
\hline Dyslipidemia & $265(49)$ & $134(33)$ & $<0.001^{\text {ब }}$ & $66(30)$ & $68(37)$ & 0.12 \\
\hline Coronary artery disease & $108(20)$ & $52(13)$ & 0.002 『 & $28(13)$ & $24(13)$ & 0.44 \\
\hline Atrial fibrillation/flutter & $158(29)$ & $54(13)$ & $<0.001^{9}$ & $27(12)$ & $27(15)$ & 0.24 \\
\hline Congestive heart failure & $42(8)$ & $27(7)$ & 0.53 & $8(4)$ & $19(10)$ & $0.01 *$ \\
\hline Cognitive disorder & $50(9)$ & $48(12)$ & 0.38 & $28(13)$ & $20(11)$ & 0.31 \\
\hline Prior ischemic stroke & $121(22)$ & $113(28)$ & 0.15 & $63(28)$ & $50(27)$ & 0.40 \\
\hline Prior ICH & $7(1.3)$ & $15(4)$ & 0.03 ף & $10(4)$ & $5(3)$ & 0.47 \\
\hline Prior TIA & $81(15)$ & $61(15)$ & 0.71 & $33(15)$ & $28(15)$ & 0.18 \\
\hline \multicolumn{7}{|c|}{ Prehospital and in-hospital characteristics } \\
\hline $\begin{array}{l}\text { Prehospital SBP, } \mathrm{mmHg} \\
(\mathrm{mean} \pm \mathrm{SD})\end{array}$ & $156.4 \pm 26.9$ & $\begin{array}{c}144.9 \pm \\
25.4\end{array}$ & $<0.001^{\text {व }}$ & $147.8 \pm 24.2$ & $141.2 \pm 26.2$ & $0.01 *$ \\
\hline $\begin{array}{l}\text { Prehospital GCS score (median, } \\
\text { IQR) }\end{array}$ & $15(3)$ & $15(1)$ & 0.12 & $15(1)$ & $15(1)$ & 0.26 \\
\hline tPA administration $(n, \%)$ & $88(16)$ & $2(0.5)$ & $<0.001^{\natural}$ & $2(1)$ & $0(0)$ & 0.22 \\
\hline Admitted to hospital (n,\%) & $435(80)$ & $161(40)$ & $<0.001^{\text {ब }}$ & $88(39)$ & $73(40)$ & 0.81 \\
\hline $\begin{array}{l}\text { Admission length, days (median, } \\
\text { IQR) }\end{array}$ & $5(18)$ & $1(5)$ & $<0.001^{ף}$ & $2(8)$ & $5(11)$ & $0.04 *$ \\
\hline
\end{tabular}

SD: standard deviation, IQR: interquartile range, ICH: intracerebral hemmorrage, TIA: transient ischemic attack, SBP: systolic blood pressure, GCS: Glasgow Coma Scale, tPA: tissue plasminogen activator.

represents $\mathrm{p}<0.05$ comparisons between strokes and stroke mimics.

$*$ represents $\mathrm{p}<0.05$ comparisons between neurological and non-neurological stroke mimics. 
Table 2. Prevalence of stroke mimic etiologies

\begin{tabular}{|l|c|c|c|}
\hline & $\begin{array}{c}\text { All stroke } \\
\text { mimics }\end{array}$ & $\begin{array}{c}\text { EMS } \\
\text { impression of } \\
\text { stroke }\end{array}$ & $\begin{array}{c}\text { EMS-other } \\
\text { impressions }\end{array}$ \\
\hline Neurological & $(\mathrm{n}=405)$ & $(\mathrm{n}=210)$ & $(\mathrm{n}=195)$ \\
\hline Non-neurological & $223(55 \%)$ & $130(62 \%)$ & $93(48 \%)$ \\
\hline Neurological mimics (n, \% of neurological mimics) & $182(45 \%)$ & $80(38 \%)$ & $102(52 \%)$ \\
\hline Seizure & $44(20 \%)$ & $28(22 \%)$ & $16(17 \%)$ \\
\hline Delirium & $23(10 \%)$ & $13(10 \%)$ & $10(11 \%)$ \\
\hline Peripheral neuropathy & $25(11 \%)$ & $14(11 \%)$ & $11(12 \%)$ \\
\hline Movement disorder & $7(3 \%)$ & $5(4 \%)$ & $2(2 \%)$ \\
\hline $\begin{array}{l}\text { Dementia (major neurocognitive } \\
\text { disorder) }\end{array}$ & $10(5 \%)$ & $7(5 \%)$ & $3(3 \%)$ \\
\hline Vertigo & $16(7 \%)$ & $5(4 \%)$ & $11(12 \%)$ \\
\hline Migraine & $42(19 \%)$ & $26(20 \%)$ & $16(17 \%)$ \\
\hline CNS neoplasm & $13(6 \%)$ & $6(5 \%)$ & $7(8 \%)$ \\
\hline Weakness & $19(9 \%)$ & $15(12 \%)$ & $4(4 \%)$ \\
\hline Non-specific symptoms & $24(11 \%)$ & $11(9 \%)$ & $13(14 \%)$ \\
\hline Non-neurological mimics (n, \% of total mimics) & $20(5 \%)$ & $14(7 \%)$ & $6(3 \%)$ \\
\hline Metabolic & $59(15 \%)$ & $21(10 \%)$ & $38(20 \%)$ \\
\hline Cardiovascular & $48(12 \%)$ & $25(12 \%)$ & $23(12 \%)$ \\
\hline Psychiatric & $36(9 \%)$ & $10(5 \%)$ & $26(13 \%)$ \\
\hline Infectious & $5(1 \%)$ & $3(1 \%)$ & $2(1 \%)$ \\
\hline Gastroenterological & $14(4 \%)$ & $7(3 \%)$ & $7(4 \%)$ \\
\hline Miscellaneous & \multicolumn{3}{|l}{} \\
\hline
\end{tabular}

EMS: Emergency Medical Services. 


\section{Figures}

Figure 1A. Stroke mimics by pathophysiological system

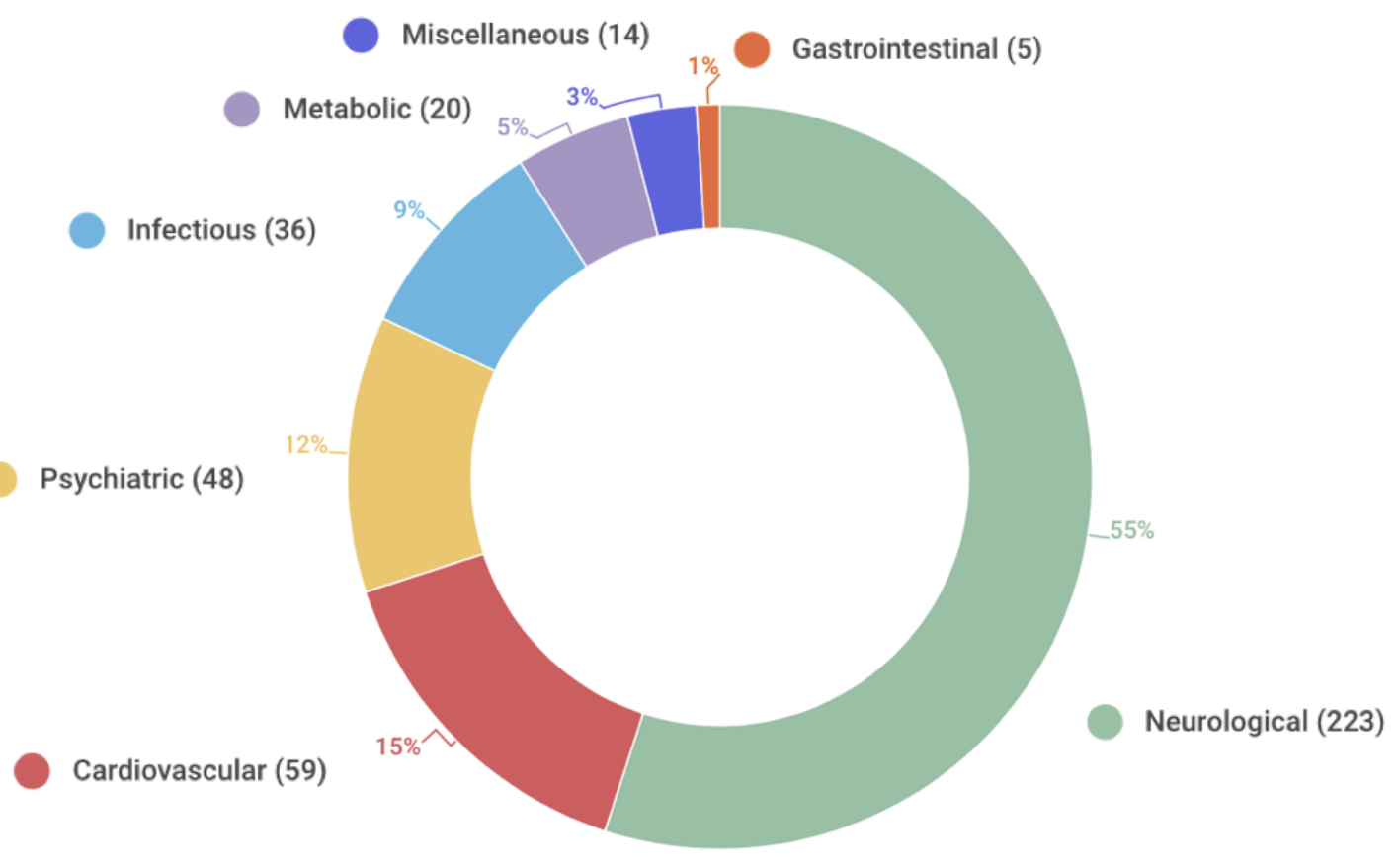

Figure 1B. Neurological stroke mimics by final diagnosis

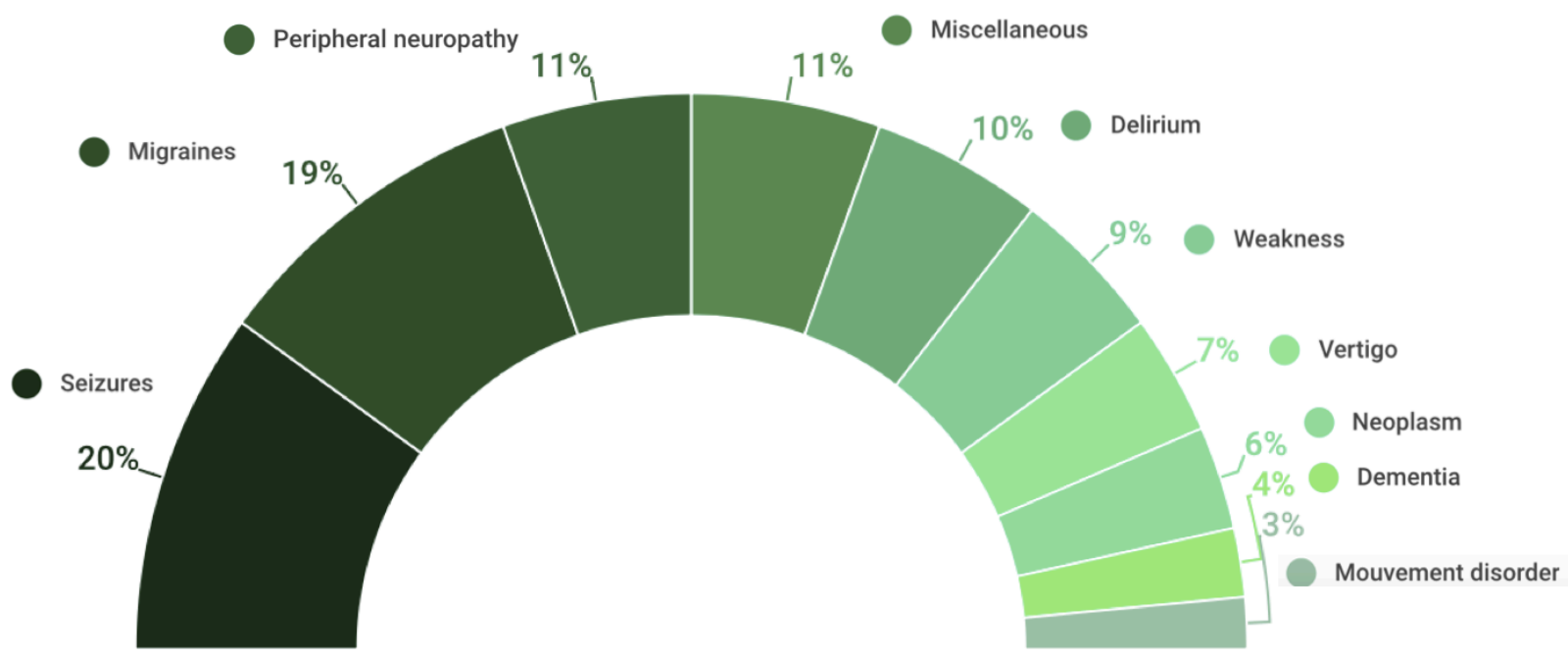

